\title{
Classification of rhizobia based on nodC and nifH gene analysis reveals a close phylogenetic relationship among Phaseolus vulgaris symbionts
}

\author{
Gisèle Laguerre, ${ }^{1}$ Sarah M. Nour, ${ }^{1}+$ Valérie Macheret, ${ }^{1}$ Juan Sanjuan, ${ }^{2}$ \\ Pascal Drouin ${ }^{1} \ddagger$ and Noëlle Amarger ${ }^{1}$
}

Author for correspondence: Gisèle Laguerre. Tel: +33 3 80693093. Fax: +33 380693224.

e-mail: laguerre@dijon.inra.fr

1 Laboratoire de

Microbiologie des Sols,

Centre de Microbiologie

du Sol et de

I'Environnement, INRA,

17 rue Sully, BP 86510 ,

F-21065 Dijon Cedex,

France

2 Departamento de Microbiología del Suelo y Sistemas Simbióticos, Estación Experimental del Zaidín, CSIC, Profesor Albareda 1, E-18008 Granada, Spain

\begin{abstract}
The nodC and nifH genes were characterized in a collection of 83 rhizobial strains which represented 23 recognized species distributed in the genera Rhizobium, Sinorhizobium, Mesorhizobium and Bradyrhizobium, as well as unclassified rhizobia from various host legumes. Conserved primers were designed from available nucleotide sequences and were able to amplify nodC and nifH fragments of about $930 \mathrm{bp}$ and $780 \mathrm{bp}$, respectively, from most of the strains investigated. RFLP analysis of the PCR products resulted in a classification of these rhizobia which was in general well-correlated with their known host range and independent of their taxonomic status. The nodC and nifH fragments were sequenced for representative strains belonging to different genera and species, most of which originated from Phaseolus vulgaris nodules. Phylogenetic trees were constructed and revealed close relationships among symbiotic genes of the Phaseolus symbionts, irrespective of their 16S-rDNA-based classification. The nodC and nifH phylogenies were generally similar, but cases of incongruence were detected, suggesting that genetic rearrangements have occurred in the course of evolution. The results support the view that lateral genetic transfer across rhizobial species and, in some instances, across Rhizobium and Sinorhizobium genera plays a role in diversification and in structuring the natural populations of rhizobia.
\end{abstract}

Keywords: Rhizobium, phylogeny, nodulation gene, nitrogen fixation gene, common bean

\section{INTRODUCTION}

The legume-nodulating bacteria, collectively called rhizobia, form a group of soil bacteria belonging to the $\alpha$-subclass of the Proteobacteria (Young \& Haukka, 1996). The current taxonomy reveals their wide diversity at the genus, species and intraspecies levels. They

\footnotetext{
†Present address: Agriculture and Agri-Food Canada, 1391 Sandford Street, London, Ont., Canada N5V 4T3.

¥Present address: Centre de Recherches et de Développement sur les Sols et les Grandes Cultures, Agriculture et Agroalimentaire Canada, 2560 Boul. Hochelaga, Sainte-Foy, Québec, Canada GIV $2 J 3$.

Abbreviation: Sym genes, symbiotic genes.

The GenBank accession numbers for the sequences reported in this paper are AF217261 through AF217272 for nodC and AF218126, AF275670 and AF275671 for $\mathrm{nifH}$.
}

are currently split into six genera: Rhizobium, Sinorhizobium, Mesorbizobium, Bradyrhizobium, Azorhizobium and the recently described genus Allorhizobium (de Lajudie et al., 1998a). These genera are phylogenetically separate from each other based on $16 \mathrm{~S}$ rDNA sequences as shown in Fig. 1, but the rhizobia do not form a coherent group since they are intermingled with other non-symbiotic bacteria (Young \& Haukka, 1996). Three species whose phylogenetic position remains unclear might constitute two other potential genera: one represented by Rhizobium galegae (Lindström, 1989) and the closely related species Rhizobium huautlense (Wang et al., 1998), and a second one by Rhizobium giardinii (Amarger et al., 1997).

Rhizobial taxonomy does not globally reflect the symbiotic features of rhizobia, particularly their host plant range. A classification of rhizobia correlated with 


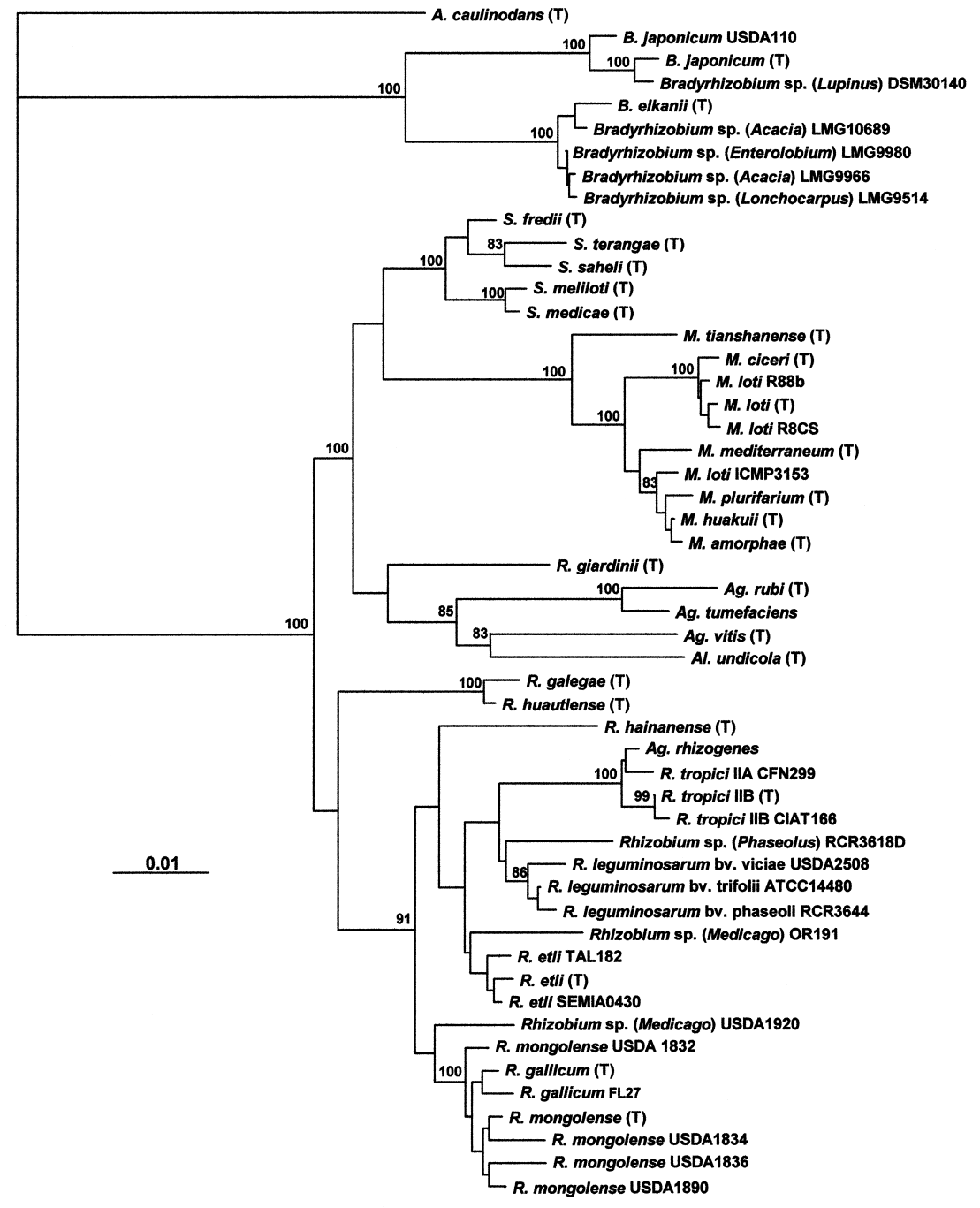

Fig. 1. Phylogenetic (neighbour-joining) tree showing the relationships between rhizobia and related agrobacteria based on $918 \mathrm{bp}$ aligned $16 \mathrm{~S}$ rDNA sequences (positions 20-937 of the nucleotide sequence of the $R$. leguminosarum bv. trifolii $16 S$ rRNA gene). Significant bootstrap probability values are indicated at the branching points (only values greater than $80 \%$ over 100 replicates are shown). This tree is almost identical to the tree based on $1450 \mathrm{bp}$ aligned 16S rDNA sequences (not shown), which was constructed by omitting the $918 \mathrm{bp}$ sequence of strain OR191. The main difference concerns the clade formed by $R$. etli, $R$. leguminosarum and $R$. tropici, which is no longer supported by significant bootstrap values in the tree including the sequence of strain OR191. The horizontal branches are drawn proportionally to the number of nucleotide substitutions per site. A., Azorhizobium; Ag., Agrobacterium; Al., Allorhizobium; B., Bradyrhizobium; M., Mesorhizobium; R., Rhizobium; S., Sinorhizobium; $(T)$, type strain. symbiotic features collides with the complexity of the molecular mechanisms involved in host specificity (for review, see Perret et al., 2000), and also the difficulty of establishing host range (Pueppke \& Broughton, 1999), particularly because of the great number of legume species. Such a classification requires a standardization of nodulation tests and the control of optimal conditions for plant growth. Although probably underestimated, it is well established that many rhizobia are able to nodulate different legume genera, and that many legumes can be nodulated by several rhizobial species. The bacterial nodulation (nod) genes, which are induced by plant flavonoids, determine the synthesis of Nod factors, the main nodulation signal molecules (for review, see Perret et al., 2000). Both the type and the amount of Nod factors are important in determining host specificity. However, rhizobia which have dissimilar nod genes and produce different Nod factors can effectively nodulate the same plant. For example, this is the case of Rhizobium etli bv. phaseoli and Rhizobium tropici, which are both nitrogen-fixing symbionts of Phaseolus vulgaris, the common bean plant (Poupot et al., 1993, 1995).
The convergence of different rhizobia that harbour distinct nod genes to the same nodulation phenotype has been reported by comparison of phylogenies inferred from housekeeping and Sym (symbiotic) gene loci (Haukka et al., 1998; Wernegreen \& Riley, 1999). On the other hand, lateral gene transfer of the Sym genes appears to be the most plausible hypothesis to explain cases of phylogenetic incongruence between Sym and housekeeping genes (Martinez-Romero \& CaballeroMellado, 1996; Young \& Haukka, 1996). Indeed, phylogenetic trees based on sequences of nod genes are generally not congruent with those based on $16 \mathrm{~S}$ rDNA sequences, but the nod trees show some correlation with host plant range (Dobert et al., 1994; Lindström et al., 1995; Ueda et al., 1995; Haukka et al., 1998; Wernegreen \& Riley, 1999). By contrast, the phylogeny of nifH genes, which encode the dinitrogenase reductase enzyme, has been reported as closely following that of $16 \mathrm{~S}$ rRNA genes (Hennecke et al., 1985; Young, 1992; Dobert et al., 1994), despite some exceptions (Eardly et al., 1992). However, these nifH phylogenies were based on analysis of a small number of sequences. Recently, Haukka et al. (1998) analysed many more sequences and 
concluded that, for rhizobia, the phylogeny of nif $H$ was generally not consistent with the phylogeny of $16 \mathrm{~S}$ rRNA, but was broadly similar to that of nodA genes. This result agrees with the fact that the nod and nif genes are often tightly linked in rhizobia, and can be located on transmissible elements such as plasmids in many rhizobial species or transposon-like elements in Mesorhizobium loti (Sullivan et al., 1995; Sullivan \& Ronson, 1998).

Although it is widely agreed that phylogenies based on stable chromosomal genes are necessary to establish a biologically meaningful rhizobial taxonomy, a proper definition of broad host range should consider the diversity of the Sym genes rather than the diversity of the species that carry them. It would thus make sense to include the characterization and the phylogenetic classification of Sym genes in the minimal standards for the description of new rhizobia as previously proposed for the 16S rRNA gene sequences (Graham et al., 1991). Such a classification should provide a complementary basic framework for our understanding of the $R \mathrm{hi}$ zobium-legume symbiosis.

The P. vulgaris microsymbionts form a taxonomically heterogeneous group. So far, five recognized species plus two distinct $16 \mathrm{~S}$ rDNA lineages have been described from rhizobial isolates recovered from bean nodules (Martinez-Romero et al., 1991; Segovia et al., 1993; van Berkum et al., 1996; Amarger et al., 1997; HerreraCervera et al., 1999). These species are distributed in two genera, Rhizobium and Sinorhizobium, and the potentially new genus represented by $R$. giardinii. Moreover, some of these species are subdivided in biovars based on the extent of host range and genetic characteristics of Sym genes (Amarger et al., 1997; Wang et al., 1999a). Our aim was to determine the congruence between classifications of rhizobia based on Sym genes and 16S rRNA and to estimate evolutionary relationships among rhizobia that have similar host legumes but are chromosomally diverse. Our main focus was the bean symbionts, but we have also analysed a larger collection of rhizobia. We initially developed a simple and rapid method to characterize Sym genes in rhizobia based on RFLP of PCR-amplified DNA, as previously achieved for 16S rRNA genes (Laguerre et al., 1994, 1997), and Sym gene loci in Rhizobium leguminosarum (Laguerre et al., 1996). Representative Sym genotypes were selected for subsequent phylogenetic analyses based on DNA sequencing. As a nodulation gene marker, we chose the nodC gene, which is a common nod gene essential for nodulation in all rhizobial species investigated so far. This gene encodes an $\mathrm{N}$-acetylglucosaminyltransferase which is involved in the first step of Nod factor assembly, and it is also a determinant of host range (reviewed by Perret et al., 2000). In addition, the nodC sequence is relatively long, which enabled the PCR amplification of large DNA fragments to a priori ensure maximum specificity of RFLP fingerprints and maximum robustness of phylogeny inferred from nucleotide sequences. As a nitrogen fixation marker, we chose the nifH gene, for which the largest number of rhizobial sequences is available for comparison (Haukka et al., 1998).

\section{METHODS}

Bacteria. The 83 strains used in this study are listed in Table 1. The bacterial sample included reference or type strains representing 23 recognized species of Rhizobium, Sinorhizobium, Mesorbizobium and Bradyrhizobium, as well as some unclassified rhizobia from various host plants. The geographic origin and the source or the references were given for most of these rhizobia in previous studies dealing with their molecular characterization based on the analysis of the 16S rRNA genes (Laguerre et al., 1994, 1997). Three additional isolates from P. vulgaris were included. Strains HT2a2 and HT4c1 are from the collection of the Laboratoire de Microbiologie des Sols in Dijon, France. They were isolated from nodules of $P$. vulgaris (cv. Vernandon) grown in pots on soil collected at the INRA-Hagetmau experimental station located in the southwest of France. There was no known history of bean cultivation and inoculation at this site. The two strains have the same $16 \mathrm{~S}$ rDNA type as Rhizobium sp. (Medicago) OR191 based on PCR-RFLP analysis (Laguerre et al., 1994, 1997; see Table 1). Sinorhizobium sp. (Phaseolus) GR-X8 was included for sequencing of nifH DNA. This strain is from the collection of the Estación Experimental del Zaidín in Granada, Spain. It was isolated from nodules of P. vulgaris (cv. Xera) and from the same soil as Sinorhizobium sp. GR-06 (Table 1). The two strains had the same $16 \mathrm{~S}$ rDNA type, which was also identical to that of Sinorhizobium fredii strains (see Table 1).

PCR amplification and restriction digestion. Approximately $930 \mathrm{bp}$ of the $1300 \mathrm{bp}$ nodC gene was amplified by using forward primers nodCF, nodCFu, nodCF2, nodCF4 or nodCFn, and reverse primer nodCI (Table 2). These primers were designed by comparing available nodC sequences for $R$. leguminosarum bv. viciae, R. tropici, R. etli bv. phaseoli, R. galegae, Sinorhizobium meliloti, S. fredii, Sinorhizobium sp. NGR234, M. loti, Bradyrhizobium japonicum and Bradyrhizobium elkanii.

The primers used for PCR amplification of about $780 \mathrm{bp}$ of the $890 \mathrm{bp}$ nifH gene were designed by comparing known nifH sequences for $R$. leguminosarum bv. trifolii, $R$. etli bv. phaseoli, S. meliloti, Sinorhizobium sp. NGR234, B. japonicum, Azorhizobium caulinodans, Azospirillum brasilense, Azotobacter chroococcum, Rhodobacter sphaeroides, Rhodobacter capsulatus and Rhodospirillum rubrum.

The cell growth conditions and the target DNA preparation were as previously described (Laguerre et al., 1997). The nodC DNA was amplified from 5-10 $\mu$ l lysed cell suspension mixed with all PCR reagents: polymerase reaction buffer (GibcoBRL); $2.5 \mathrm{mM} \mathrm{MgCl}_{2} ; 200 \mu \mathrm{M}$ (each) dATP, dCTP, dTTP, dGTP; $0 \cdot 4-0.8 \mu \mathrm{M}$ (each) nodC primers; $0.04 \mathrm{U}$ Taq DNA polymerase (Gibco-BRL) $\mu \mathrm{l}^{-1}$. DNA amplification was done by using a standard temperature profile including an annealing temperature of $55^{\circ} \mathrm{C}$ (Laguerre et al., 1994). The procedure for amplification of the nifH fragments was similar, except that reactions were made with $1.5 \mathrm{mM} \mathrm{MgCl}_{2}, 20 \mu \mathrm{M}$ (each) $\mathrm{dNTP}$ and $0 \cdot 1 \mu \mathrm{M}$ (each) nifH primers and the annealing temperature was increased to $57^{\circ} \mathrm{C}$. For a few strains, multiple nodC bands were obtained. In these cases, a small piece of agarose containing the band of the expected size was aspirated by using a hypodermic needle and used as a template in a new PCR.

Restriction pattern analysis of the PCR products with the 
Table 1. Strains used in this study and data of PCR-RFLP analysis of nodC and nifH gene fragments

The data are displayed as restriction patterns encoded by letters. Identical patterns are designated by the same letter(s) for each endonuclease; the on-line version of this paper (available at http://mic.sgmjournals.org) contains a supplementary table giving information on the size of the restriction fragments for each pattern. ND, Not determined; No, unsuccessful PCR amplification. ${ }^{\mathrm{T}}$, Type strain of the species.

\begin{tabular}{|c|c|c|c|c|c|c|c|c|c|c|c|c|c|c|c|}
\hline \multirow[t]{2}{*}{ Strain } & \multirow[t]{2}{*}{$\begin{array}{c}\text { Species or } \\
16 \text { S rDNA type* }\end{array}$} & \multirow[t]{2}{*}{ Biovar $†$} & \multirow[t]{2}{*}{ Plant of origin } & \multirow[t]{2}{*}{$\begin{array}{l}\text { nod } \\
\text { type }\end{array}$} & \multirow[t]{2}{*}{$\begin{array}{c}n i f \\
\text { type } \neq\end{array}$} & \multicolumn{6}{|c|}{ nod $C$ restriction pattern } & \multicolumn{3}{|c|}{$\begin{array}{l}\text { nifH restriction } \\
\text { pattern }\end{array}$} & \multirow[t]{2}{*}{ Source or reference $\$$} \\
\hline & & & & & & $C f o \mathrm{I}$ & HaeIII & HinfI & MspI & NdeII & $R s a \mathrm{I}$ & HaeIII & I $M s p I$ & NdeII & \\
\hline USDA $2370^{\mathrm{T}}$ & R. leguminosarum & viciae & Pisum sativum & $\mathrm{V} 1$ & $\mathrm{~V} 1$ & A & A & A & A & A & A & A & A & A & Laguerre et al. (1997) \\
\hline JISP24 & R. leguminosarum & viciae & Pisum sativum & $\mathrm{V} 2$ & V 2 & A & $\mathrm{A}$ & $\mathrm{A}$ & B & $\mathrm{A}$ & $\mathrm{A}$ & B & B & B & Laguerre et al. (1994) \\
\hline F5NP1E & R. leguminosarum & viciae & Pisum sativum & V 3 & ND & B & B & A & A & A & B & ND & ND & ND & MSDJ \\
\hline L113 & R. leguminosarum & viciae & Lens esculenta & V 4 & V 3 & A & A & B & A & A & A & B & $\mathrm{C}$ & $\mathrm{A}$ & Laguerre et al. (1994) \\
\hline 8401 & R. leguminosarum & viciae & & V 5 & $\mathrm{~V} 4$ & $\mathrm{C}$ & $\mathrm{C}$ & A & A & B & $\mathrm{C}$ & $\mathrm{C}$ & B & $\mathrm{C}$ & Laguerre et al. (1994) \\
\hline USDA 2071 & R. leguminosarum & trifolii & Trifolium repens & Т 1 & $\mathrm{~T} 1$ & $\mathrm{D}$ & $\mathrm{D}$ & $\mathrm{C}$ & $\mathrm{C}$ & $\mathrm{C}$ & $\mathrm{D}$ & $\mathrm{D}$ & $\mathrm{D}$ & $\mathrm{D}$ & Laguerre et al. (1997) \\
\hline $\operatorname{cc} 2480 \mathrm{a}$ & R. leguminosarum & trifolii & Trifolium subterraneum & Т 2 & No & $\mathrm{D}$ & $\mathrm{E}$ & $\mathrm{D}$ & $\mathrm{D}$ & $\mathrm{D}$ & $\mathrm{E}$ & No & No & No & Laguerre et al. (1994) \\
\hline T143 & R. leguminosarum & trifolii & Trifolium pratense & Т 3 & Т 2 & $\mathrm{E}$ & $\mathrm{F}$ & $\mathrm{D}$ & $\mathrm{E}$ & $\mathrm{E}$ & $\mathrm{F}$ & $\mathrm{E}$ & $\mathrm{E}$ & E & Laguerre et al. (1994) \\
\hline H112 & R. leguminosarum & trifolii & Phaseolus vulgaris & $\mathrm{T} 1$ & Т 3 & $\mathrm{D}$ & $\mathrm{D}$ & $\mathrm{C}$ & $\mathrm{C}$ & $\mathrm{C}$ & $\mathrm{D}$ & $\mathrm{F}$ & $\mathrm{D}$ & $\mathrm{F}$ & Laguerre et al. (1994) \\
\hline H132 & R. leguminosarum & phaseoli & Phaseolus vulgaris & P 1 & P 1 & $\mathrm{~F}$ & G & $\mathrm{E}$ & $\mathrm{F}$ & $\mathrm{F}$ & G & G & $\mathrm{F}$ & G & Laguerre et al. (1997) \\
\hline $\mathrm{H} 251$ & R. giardinii & phaseoli & Phaseolus vulgaris & P 1 & ND & $\mathrm{F}$ & G & $\mathrm{E}$ & $\mathrm{F}$ & $\mathrm{F}$ & G & ND & ND & ND & Laguerre et al. (1994) \\
\hline RCR3618D & $\begin{array}{l}\text { Type Rhizobium sp. } \\
\text { RCR3618D }\end{array}$ & 'phaseoli' & Phaseolus vulgaris & P 1 & ND & $\mathrm{F}$ & G & $\mathrm{E}$ & $\mathrm{ND}$ & ND & G & ND & ND & $\mathrm{ND}$ & van Berkum et al. (1996) \\
\hline VikingI & R. etli & phaseoli & Phaseolus vulgaris & P 2 & P 1 & $\mathrm{~F}$ & G & $\mathrm{E}$ & G & $\mathrm{F}$ & G & G & $\mathrm{F}$ & G & Laguerre et al. (1994) \\
\hline PhD12 & R. gallicum & phaseoli & Phaseolus vulgaris & P 3 & P 1 & $\mathrm{~F}$ & G & $\mathrm{E}$ & G & G & G & G & $\mathrm{F}$ & G & Laguerre et al. (1994) \\
\hline H441 & R. leguminosarum & phaseoli & Phaseolus vulgaris & P 4 & P 1 & G & G & $\mathrm{F}$ & $\mathrm{H}$ & $\mathrm{H}$ & $\mathrm{H}$ & G & $\mathrm{F}$ & G & Laguerre et al. (1994) \\
\hline CFN $42^{\mathrm{T}}$ & R. etli & phaseoli & Phaseolus vulgaris & P 4 & P 1 & G & G & $\mathrm{F}$ & $\mathrm{H}$ & $\mathrm{H}$ & $\mathrm{H}$ & G & $\mathrm{F}$ & G & Laguerre et al. (1997) \\
\hline CFN 299 & R. tropici-IIA & & Phaseolus vulgaris & $\operatorname{Tr} 1$ & $\operatorname{Tr} 1$ & $\mathrm{H}$ & $\mathrm{H}$ & B & I & I & I & $\mathrm{H}$ & G & $\mathrm{H}$ & Laguerre et al. (1997) \\
\hline Airel 8, Airel 18 & R. tropici-IIA & & Phaseolus vulgaris & $\operatorname{Tr} 1$ & $\operatorname{Tr} 1$ & $\mathrm{H}$ & $\mathrm{H}$ & B & I & I & I & $\mathrm{H}$ & G & $\mathrm{H}$ & Amarger et al. (1994) \\
\hline CIAT $899^{\mathrm{T}}$ & R. tropici-IIB & & Phaseolus vulgaris & $\operatorname{Tr} 2$ & $\operatorname{Tr} 1$ & I & $\mathrm{H}$ & B & I & I & $\mathrm{J}$ & $\mathrm{H}$ & G & $\mathrm{H}$ & Laguerre et al. (1997) \\
\hline BR859 & R. tropici-IIB & & Phaseolus vulgaris & $\operatorname{Tr} 2$ & $\operatorname{Tr} 1$ & I & $\mathrm{H}$ & B & I & I & $\mathrm{J}$ & $\mathrm{H}$ & G & $\mathrm{H}$ & Laguerre et al. (1994) \\
\hline BR864 & R. tropici & & Leucaena leucocephala & $\operatorname{Tr} 2$ & $\operatorname{Tr} 2$ & I & $\mathrm{H}$ & B & I & I & $\mathrm{J}$ & $\mathrm{H}$ & G & I & Laguerre et al. (1994) \\
\hline HT2a2, HT4c1 & $\begin{array}{l}\text { Type Rhizobium sp. } \\
\text { OR191 }\end{array}$ & & Phaseolus vulgaris & $\operatorname{Tr} 1$ & ND & $\mathrm{H}$ & $\mathrm{H}$ & B & I & I & I & ND & ND & $\mathrm{ND}$ & MSDJ \\
\hline $\begin{array}{l}\text { R602sp }{ }^{\mathrm{T}}, \mathrm{PhF} 29, \\
\text { PhP222 }\end{array}$ & R. gallicum & gallicum & Phaseolus vulgaris & G 1 & G 1 & $\mathrm{~J}$ & I & G & $\mathrm{J}$ & $\mathrm{J}$ & K & I & $\mathrm{H}$ & $\mathrm{J}$ & $\begin{array}{l}\text { Laguerre et al. (1994); Amarger } \\
\text { et al. (1997) }\end{array}$ \\
\hline F127 & R. gallicum & gallicum & Phaseolus vulgaris & G 2 & G 2 & $\mathrm{~J}$ & $\mathrm{~J}$ & $\mathrm{H}$ & K & K & $\mathrm{L}$ & $\mathrm{J}$ & $\mathrm{H}$ & K & Laguerre et al. (1994) \\
\hline USDA 3497 & Type R. mongolense & 'gallicum' & Leucaena leucocephala & G 3 & G 2 & $\mathrm{~J}$ & I & G & $\mathrm{L}$ & $\mathrm{J}$ & M & $\mathrm{J}$ & $\mathrm{H}$ & K & Laguerre et al. (1997) \\
\hline $\mathrm{H} 152^{\mathrm{T}}$ & R. giardinii & giardinii & Phaseolus vulgaris & Gi 1 & No & $\mathrm{K}$ & $\mathrm{K}$ & $\mathrm{H}$ & $\mathrm{M}$ & $\mathrm{L}$ & $\mathrm{N}$ & No & No & No & Laguerre et al. (1994) \\
\hline $\mathrm{H} 241$ & R. giardinii & giardinii & Phaseolus vulgaris & Gi 1 & ND & K & K & $\mathrm{H}$ & M & $\mathrm{L}$ & $\mathrm{N}$ & ND & ND & ND & Laguerre et al. (1994) \\
\hline GR-06 & Type $S$. fredii & & Phaseolus vulgaris & Sp 1 & Sp 1 & $\mathrm{~L}$ & $\mathrm{~L}$ & I & $\mathrm{N}$ & M & $\mathrm{O}$ & K & I & $\mathrm{L}$ & $\begin{array}{l}\text { Herrera-Cervera et al. } \\
\text { (1999) }\end{array}$ \\
\hline GR-X8 & Type $S$. fredii & & Phaseolus vulgaris & ND & ND & ND & ND & ND & $\mathrm{ND}$ & $\mathrm{ND}$ & ND & ND & $\mathrm{ND}$ & $\mathrm{ND}$ & EEZ \\
\hline USDA $1002^{\mathrm{T}}$ & S. meliloti & & Medicago sativa & Me 1 & Me 1 & M & M & $\mathrm{J}$ & $\mathrm{O}$ & $\mathrm{N}$ & $\mathrm{P}$ & $\mathrm{L}$ & $\mathrm{J}$ & M & Laguerre et al. (1997) \\
\hline RCR2011 & S. meliloti & & Medicago sativa & Me 2 & Me 2 & M & $\mathrm{N}$ & $\mathrm{K}$ & $\mathrm{P}$ & $\mathrm{O}$ & Q & $\mathrm{L}$ & K & M & Laguerre et al. (1994) \\
\hline OR191 & $\begin{array}{l}\text { Type Rhizobium sp. } \\
\text { OR191 }\end{array}$ & & Medicago sativa & Me 3 & Me 3 & $\mathrm{~N}$ & $\mathrm{O}$ & $\mathrm{L}$ & $\mathrm{P}$ & $\mathrm{P}$ & $\mathrm{R}$ & M & $\mathrm{L}$ & $\mathrm{N}$ & Laguerre et al. (1997) \\
\hline M1 & S. medicae & & Medicago orbicularis & Me 4 & Me 4 & $\mathrm{O}$ & $\mathrm{P}$ & M & $\mathrm{P}$ & $\mathrm{N}$ & S & $\mathrm{N}$ & M & $\mathrm{O}$ & Laguerre et al. (1997) \\
\hline M102 & S. medicae & & Medicago truncatula & Me 4 & $\mathrm{Me} 4$ & $\mathrm{O}$ & $P$ & $\mathrm{M}$ & $\mathrm{P}$ & $\mathrm{N}$ & S & $\mathrm{N}$ & M & $\mathrm{O}$ & Laguerre et al. (1997) \\
\hline $\begin{array}{l}\text { USDA } 1844^{\mathrm{T}}, \\
\text { USDA } 1890\end{array}$ & R. mongolense & & Medicago rustica & Me 5 & Me 5 & $\mathrm{P}$ & $\mathrm{O}$ & $\mathrm{L}$ & $\mathrm{P}$ & Q & $\mathrm{R}$ & $\mathrm{O}$ & $\mathrm{N}$ & $\mathrm{P}$ & van Berkum et al. (1998) \\
\hline NZP $2213^{\mathrm{T}}$ & M. loti & & Lotus tenuis & Lo 1 & Lo 1 & Q & Q & $\mathrm{N}$ & Q & $\mathrm{R}$ & $\mathrm{T}$ & $\mathrm{P}$ & $\mathrm{O}$ & Q & Laguerre et al. (1997) \\
\hline NZP 2037 & M. loti & & Lotus divaricatus & Lo 2 & Lo 2 & $\mathrm{R}$ & $\mathrm{Q}$ & $\mathrm{O}$ & $\mathrm{Q}$ & $S$ & $\mathrm{U}$ & $\mathrm{P}$ & $\mathrm{O}$ & $\mathrm{R}$ & Laguerre et al. (1997) \\
\hline NZP 2234 & M. loti & & Lotus cornitulatus & Lo 3 & Lo 3 & S & $\mathrm{Q}$ & M & $\mathrm{R}$ & $\mathrm{T}$ & $\mathrm{T}$ & Q & $P$ & S & Laguerre et al. (1997) \\
\hline MSDJ 865 & M. loti & & Lotus cornitulatus & Lo 4 & Lo 4 & S & Q & $\mathrm{P}$ & Q & S & $\mathrm{T}$ & $\mathrm{P}$ & $\mathrm{P}$ & S & Laguerre et al. (1997) \\
\hline $\mathrm{A}-1 \mathrm{BS}^{\mathrm{T}}$ & M. tianshanense & & Glycyrrbiza pallidiflora & Ti 1 & Ti 1 & $\mathrm{~T}$ & $\mathrm{R}$ & $\mathrm{Q}$ & $\mathrm{s}$ & $\mathrm{U}$ & V & $\mathrm{R}$ & Q & $\mathrm{T}$ & Chen et al. (1995) \\
\hline $\begin{array}{l}\text { UPM-Ca } 7^{\mathrm{T}} \\
\text { USDA } 3233\end{array}$ & M. ciceri & & Cicer arietinum & Ci 1 & Ci 1 & $\mathrm{U}$ & $\mathrm{S}$ & $\mathrm{R}$ & $\mathrm{T}$ & V & W & S & $\mathrm{O}$ & $\mathrm{U}$ & Laguerre et al. (1997) \\
\hline UPMCa $36^{\mathrm{T}}$ & M. mediterraneum & & Cicer arietinum & $\mathrm{Ci} 2$ & Ci 1 & $\mathrm{U}$ & s & S & $\mathrm{T}$ & W & $\mathrm{W}$ & S & $\mathrm{O}$ & U & Laguerre et al. (1997) \\
\hline MSDJ 2184 & $\begin{array}{l}\text { Type Mesorhizobium sp. } \\
\text { MSDJ } 2184\end{array}$ & & Coronilla varia & Co 1 & Co 1 & $\mathrm{~V}$ & $\mathrm{~T}$ & $\mathrm{~T}$ & $\mathrm{U}$ & $\mathrm{x}$ & $\mathrm{x}$ & ND & $\mathrm{R}$ & V & Laguerre et al. (1997) \\
\hline $7653 \mathrm{R}$ & M. buakuii & & Astragalus sinicus & As 1 & As 1 & W & $\mathrm{U}$ & $\mathrm{U}$ & V & Y & $\mathrm{Y}$ & $\mathrm{T}$ & S & $\mathrm{T}$ & Yang et al. (1999) \\
\hline $\operatorname{Ra} 5$ & M. buakuii & & Astragalus sinicus & As 1 & As 2 & ND & ND & $\mathrm{U}$ & $\mathrm{V}$ & ND & ND & $\mathrm{U}$ & S & $\mathrm{T}$ & Yang et al. (1999) \\
\hline ACCC $19665^{\mathrm{T}}$ & M. amorphae & & Amorpha fruticosa & Am 1 & Am 1 & $\mathrm{X}$ & $\mathrm{V}$ & $\mathrm{E}$ & W & $\mathrm{Z}$ & W & $\mathrm{V}$ & $\mathrm{T}$ & W & Wang et al. (1999b) \\
\hline HL56, SH15003 & M. amorphae & & Amorpha fruticosa & ND & Am 1 & ND & ND & ND & ND & ND & ND & $\mathrm{V}$ & $\mathrm{T}$ & W & Wang et al. (1999b) \\
\hline $\begin{array}{l}\text { ORS } 1004, \text { ORS } \\
1010\end{array}$ & M. plurifarium & & Acacia senegal & MP 1 & MP 1 & $\mathrm{Y}$ & W & $\mathrm{V}$ & $\mathrm{X}$ & AA & $\mathrm{Z}$ & W & I & $\mathrm{X}$ & de Lajudie et al. (1998b) \\
\hline $\begin{array}{l}\text { ORS } 1009^{\mathrm{T}} \\
\text { ORS } 1007\end{array}$ & S. terangae & acaciae & Acacia laeta & Ac 1 & Ac 1 & $\mathrm{Z}$ & $\mathrm{x}$ & W & Y & $\mathrm{AB}$ & AA & $\mathrm{x}$ & $\mathrm{U}$ & Y & $\begin{array}{l}\text { de Lajudie et al. (1994); } \\
\text { Laguerre et al. (1997) }\end{array}$ \\
\hline ORS 51 & S. terangae & sesbaniae & Sesbania rostrata & No & Se 1 & No & No & No & No & No & No & Y & $\mathrm{V}$ & z & de Lajudie et al. (1994) \\
\hline ORS $609^{\mathrm{T}}$ & S. sabeli & sesbaniae & Sesbania cannabina & No & Se 2 & No & No & No & No & No & No & $\mathrm{Z}$ & $\mathrm{V}$ & $\mathrm{Z}$ & Laguerre et al. (1997) \\
\hline ORS 611 & S. sabeli & sesbaniae & Sesbania grandiflora & No & Se 2 & No & No & No & No & No & No & $\mathrm{Z}$ & $\mathrm{V}$ & $\mathrm{Z}$ & de Lajudie et al. (1994) \\
\hline So $2^{\mathrm{T}}$, S 35 & R. huautlense & & Sesbania herbacea & Se 1 & Se 3 & $\mathrm{AA}$ & $\mathrm{Y}$ & $\mathrm{X}$ & $\mathrm{Z}$ & $\mathrm{AC}$ & $\mathrm{AB}$ & $\mathrm{Y}$ & V & $\mathrm{AA}$ & Wang et al. (1998) \\
\hline HAMBI $540^{\mathrm{T}}$ & R. galegae & & Galega orientalis & Ga 1 & Ga 1 & $\mathrm{AB}$ & $\mathrm{Z}$ & Y & $\mathrm{AA}$ & $\mathrm{AD}$ & $\mathrm{AC}$ & $\mathrm{AA}$ & $\mathrm{W}$ & $\mathrm{AB}$ & Laguerre et al. (1997) \\
\hline USDA $205^{\mathrm{T}}$ & S. fredii & & Glycine $\max$ & F 1 & F 1 & $\mathrm{~T}$ & AA & Z & $\mathrm{AB}$ & $\mathrm{AE}$ & $\mathrm{AD}$ & $\mathrm{AB}$ & $\mathrm{X}$ & $\mathrm{AC}$ & Laguerre et al. (1997) \\
\hline MSDJ 1536 & S. fredii & & Glycine $\max$ & F 1 & F 1 & $\mathrm{~T}$ & $\mathrm{AA}$ & $\mathrm{Z}$ & $\mathrm{AB}$ & $\mathrm{AE}$ & $\mathrm{AD}$ & $\mathrm{AB}$ & $\mathrm{X}$ & $\mathrm{AC}$ & Laguerre et al. (1994) \\
\hline MSDJ 1537 & S. fredii & & Glycine max & F 1 & F 1 & $\mathrm{~T}$ & $\mathrm{AA}$ & Z & $\mathrm{AB}$ & $\mathrm{AE}$ & $\mathrm{AD}$ & $\mathrm{AB}$ & $\mathrm{X}$ & ND & Laguerre et al. (1994) \\
\hline
\end{tabular}


Table 1 (cont.)

\begin{tabular}{|c|c|c|c|c|c|c|c|c|c|c|c|c|c|c|c|}
\hline \multirow[t]{2}{*}{ Strain } & \multirow[t]{2}{*}{$\begin{array}{c}\text { Species or } \\
\text { 16S rDNA type* }\end{array}$} & \multirow[t]{2}{*}{ Biovart } & \multirow[t]{2}{*}{ Plant of origin } & \multirow[t]{2}{*}{$\begin{array}{l}\text { nod } \\
\text { type } \ddagger\end{array}$} & \multirow[t]{2}{*}{$\begin{array}{c}n i f \\
\text { type }\end{array}$} & \multicolumn{6}{|c|}{ nod $C$ restriction pattern } & \multicolumn{3}{|c|}{$\begin{array}{c}\text { nifH restriction } \\
\text { pattern }\end{array}$} & \multirow[t]{2}{*}{ Source or reference $\$$} \\
\hline & & & & & & $C f_{0} \mathrm{I}$ & HaeIII & HinfI & MspI & NdeII & RsaI & HaeIII & MspI & NdeII & \\
\hline $\begin{array}{l}\text { USDA } 6^{\mathrm{T}}, \\
\text { USDA } 110\end{array}$ & B. japonicum & & Glycine $\max$ & $\mathrm{J} 1$ & $\mathrm{~J} 1$ & $\mathrm{AC}$ & $\mathrm{AB}$ & $\mathrm{AA}$ & $\mathrm{AC}$ & $\mathrm{AF}$ & $\mathrm{AE}$ & $\mathrm{AC}$ & Y & $\mathrm{AD}$ & Laguerre et al. (1997) \\
\hline USDA 123 & B. japonicum & & Glycine $\max$ & $\mathrm{J} 1$ & $\mathrm{~J} 1$ & $\mathrm{AC}$ & $\mathrm{AB}$ & $\mathrm{AA}$ & $\mathrm{AC}$ & $\mathrm{AF}$ & $\mathrm{AE}$ & $\mathrm{AC}$ & Y & $\mathrm{AD}$ & USDA \\
\hline G49 & B. japonicum & & Glycine $\max$ & $\mathrm{J} 1$ & $\mathrm{~J} 1$ & $\mathrm{AC}$ & $\mathrm{AB}$ & $\mathrm{AA}$ & $\mathrm{AC}$ & $\mathrm{AF}$ & $\mathrm{AE}$ & AC & Y & $\mathrm{AD}$ & Lagacherie et al. (1997) \\
\hline USDA 138 & B. japonicum & & Glycine $\max$ & $\mathrm{J} 1$ & $\mathrm{~J} 1$ & $\mathrm{AC}$ & $\mathrm{AB}$ & $\mathrm{AA}$ & $\mathrm{ND}$ & ND & $\mathrm{AE}$ & $\mathrm{AC}$ & Y & $\mathrm{AD}$ & USDA \\
\hline 2281 & B. liaoningense & & Glycine max & $\mathrm{J} 1$ & $\mathrm{~J} 1$ & $\mathrm{AC}$ & $\mathrm{AB}$ & AA & $\mathrm{AC}$ & $\mathrm{AF}$ & $\mathrm{AE}$ & $\mathrm{AC}$ & Y & $\mathrm{AD}$ & Xu et al. (1995) \\
\hline USDA $76^{\mathrm{T}}$ & B. elkanii & & Glycine $\max$ & E 1 & E 1 & $\mathrm{AD}$ & $\mathrm{AC}$ & M & $\mathrm{AD}$ & AG & $\mathrm{AE}$ & $\mathrm{AD}$ & Z & $\mathrm{AE}$ & Laguerre et al. (1997) \\
\hline $\begin{array}{l}\text { USDA } 61, \text { USDA } \\
94, \text { USDA } 340\end{array}$ & B. elkanii & & Glycine max & ND & E 1 & ND & ND & ND & ND & ND & ND & $\mathrm{AD}$ & $\mathrm{Z}$ & $\mathrm{AE}$ & $\begin{array}{c}\text { Kuykendall et al. (1992); } \\
\text { Laguerre et al. (1997) }\end{array}$ \\
\hline CB756 & $\begin{array}{l}\text { Type Bradyrhizobium sp. } \\
\text { MSDJ } 718\end{array}$ & & Macrotyloma africanum & Ma 1 & Ma 1 & $\mathrm{AE}$ & $\mathrm{AB}$ & M & $\mathrm{AE}$ & $\mathrm{AH}$ & $\mathrm{AF}$ & $\mathrm{AE}$ & AA & $\mathrm{AF}$ & Laguerre et al. (1994) \\
\hline MSDJ 718 & $\begin{array}{l}\text { Type Bradyrhizobium sp. } \\
\text { MSDJ } 718\end{array}$ & & Lupinus luteus & Lu 1 & Lu 1 & $\mathrm{AF}$ & $\mathrm{AD}$ & $\mathrm{AB}$ & $\mathrm{AF}$ & AI & $\mathrm{AG}$ & $\mathrm{AF}$ & $\mathrm{AB}$ & AG & Laguerre et al. (1997) \\
\hline LB84 & Bradyrhizobium sp. & & Lupinus albus & Lu 2 & Lu 2 & AG & $\mathrm{AD}$ & $\mathrm{AC}$ & AG & AJ & AG & $\mathrm{AF}$ & $\mathrm{AB}$ & $\mathrm{AH}$ & Lagacherie et al. (1983) \\
\hline LB86 & Bradyrhizobium sp. & & Lupinus albus & ND & Lu 2 & ND & ND & ND & ND & ND & ND & $\mathrm{AF}$ & $\mathrm{AB}$ & $\mathrm{AH}$ & Lagacherie et al. (1983) \\
\hline VK4 & $\begin{array}{l}\text { Type Bradyrhizobium sp. } \\
\text { VK4 }\end{array}$ & & Lupinus sp. & ND & Lu 3 & ND & ND & ND & ND & ND & ND & $\mathrm{AF}$ & AC & $\mathrm{AH}$ & Bergersen et al. (1986) \\
\hline WU425 & $\begin{array}{l}\text { Type Bradyrhizobium sp. } \\
\text { VK4 }\end{array}$ & & Ornithopus compressus & Lu 3 & Lu 3 & $\mathrm{AH}$ & $\mathrm{AE}$ & $\mathrm{AD}$ & $\mathrm{AH}$ & $\mathrm{AK}$ & $\mathrm{AH}$ & $\mathrm{AF}$ & $\mathrm{AC}$ & $\mathrm{AH}$ & Bergersen et al. (1986) \\
\hline
\end{tabular}

* The 16S rDNA types were characterized by RFLP analysis of PCR-amplified 16S rRNA genes as previously reported (Laguerre et al., 1994, 1997).

† The biovars indicated within quotation marks were identified in this study on the basis of the analysis of the Sym genes.

$\ddagger$ The $\operatorname{nod} \mathrm{C}$ and nifH types represent the combination of the restriction patterns; types sharing identical restriction patterns were given the same letter as follows: V, bv. viciae; T, bv. trifolii; P, bv. phaseoli; Tr, tropici; G, gallicum; Gi, giardinii; Me, Medicago; Lo, Lotus; Ti, tianshanense; Ci, Cicer; Co, Coronilla; As, Astragalus; Am, amorphae; Mp, M. plurifarium; Ac, bv. acaciae; Se, Sesbania; Ga, Galega; F, fredii; J, japonicum; E, elkanii; Ma, Macrotyloma; Lu, Lupinus. ND, Not determined; No, unsuccessful PCR amplification. SUSDA, Rhizobium culture collection, Beltsville Agricultural Research Center, Beltsville, MD, USA; MSDJ, Laboratoire de Microbiologie des Sols, INRA, Dijon, France; EEZ, Estación Experimental del Zaidín, CSIC, Granada, Spain.

Table 2. Oligonucleotides used as PCR or sequencing primers

\begin{tabular}{|llc|}
\hline Primer & \multicolumn{1}{c|}{$\mathbf{5}^{\prime} \mathbf{- 3}^{\prime}$ nucleotide sequence* } & Position $\boldsymbol{~}$ \\
\hline nodCF & AYGTHGTYGAYGACGGTTC & $251-269$ \\
nodCFu & AYGTHGTYGAYGACGGITC & $251-269$ \\
nodCF2 & AYGTHGTYGAYGACGGCTC & $251-269$ \\
nodCF4 & AYGTHGTYGAYGACGGATC & $251-269$ \\
nodCFn & AGGTGGTYGAYGACGGTTC & $251-269$ \\
nodCI & CGYGACAGCCANTCKCTATTG & $1160-1181$ \\
nifHF & TACGGNAARGGSGGNATCGGCAA & $25-50$ \\
nifHI & AGCATGTCYTCSAGYTCNTCCA & $787-808$ \\
\hline
\end{tabular}

$* \mathrm{Y}=\mathrm{C}$ or $\mathrm{T} ; \mathrm{H}=\mathrm{A}, \mathrm{C}$ or $\mathrm{T} ; \mathrm{R}=\mathrm{A}$ or $\mathrm{G} ; \mathrm{S}=\mathrm{C}$ or $\mathrm{G} ; \mathrm{K}=\mathrm{G}$ or $\mathrm{T} ; \mathrm{N}=\mathrm{A}, \mathrm{C}, \mathrm{G}$ or $\mathrm{T} ; \mathrm{I}=$ inosine.

†Position of the primer nucleotide sequences in the corresponding nod $C$ sequence of $R$. leguminosarum bv. viciae and nifH sequence of R. leguminosarum bv. trifolii.

restriction endonucleases listed in Table 1 was as previously described (Laguerre et al., 1997).

Sequencing of nodC and nifH DNA. All the nucleotide sequences, apart from two nifH sequences (see below), obtained in this study were determined by Genome Express (Grenoble, France). In a first attempt, crude nodC PCR products were directly sequenced on both strands by using primers nodCF and nodCI. The less degenerate primer nodCFn, which matched the same oligonucleotide sequence as nodCF, was used for PCR amplification and sequencing of crude PCR products from strains PhD12 and H251. Sequencing of nodC DNA with nodCFn and nodCI was achieved for strains H132 and Viking I only after purification of PCR products by either $70 \%$ ethanol precipitation in the presence of $0.7 \mathrm{M}$ ammonium acetate or (when multiple PCR products were obtained) extraction of the nodC fragment from agarose gels by using a QIAEX II gel extraction kit (Qiagen) followed by ethanol/ammonium acetate precipitation. The sequences of the nifH fragments and the nodC fragment of strains R602sp, H152, ACCC 19665, USDA 2071 and OR191 were obtained after cloning the PCR products by using either a pT7Blue T-Vector kit (Novagen), a pGEM-T Vector kit (Promega) or a TOPO TA cloning kit (Invitrogen) according to the manufacturers' instructions. The result of cloning was checked by PCR amplification by using the vector plasmid primers T7 and SP6 according to the procedure described by Novagen. The crude PCR products were directly used for sequencing with both primers T7 and SP6. Sequencing of nifH fragments of strains GR-06 and GR-X8 was done in an ABI 373 XL Stretch Sequencer (Perkin-Elmer Biosystems) using an ABI Prism BigDye Terminator kit and vector-based sequencing primers (M13 universal and reverse primers). 
From all these experiments, 450-750 nucleotides of each DNA strand were determined and an 808-935 bp sequence of nodC fragments and a 736-783 bp sequence of the nif $H$ gene were reconstituted for each strain. Restriction site analyses of the sequences were performed by using the Bisance software (Dessen et al., 1990).

Phylogenetic analysis. The sequences have been deposited in the GenBank database under accession numbers AF217261 through AF217272 for the nodC sequences and AF218126, AF275670 and AF275671 for the nifH sequences of strains R602sp, GR-X8 and GR-06, respectively.

The accession numbers of the published sequences used for comparisons were as follows. The numbers for the nodC sequences were: M13658 (R. leguminosarum bv. viciae 238), X98514 (R. tropici IIA CFN 299), X87578 (R. galegae HAMBI 1174), M11268 (S. meliloti 1021), M73699 (S. fredii USDA 257), X73362 (Sinorhizobium sp. NGR234), X52958 (M. loti NZP 2037), U53327 [Mesorhizobium sp. (Oxytropis) N33], AF105431 (Bradyrhizobium sp. SNU001), L18897 (Azorhizobium caulinodans ORS $\left.571^{\mathrm{T}}\right)$. The numbers for the $n$ if $H$ sequences were: K00490 ( $R$. leguminosarum bv. trifolii SU329), M55225 (R. tropici IIB CIAT 899 ${ }^{\mathrm{T}}$ ), M15942 (R. etli bv. phaseoli CFN 42 ${ }^{\mathrm{T}}$ ), M55227 (R. etli bv. phaseoli Olivia-4), AF107621 (R. etli bv. mimosae Mim2), M55226 (Rhizobium gallicum bv. gallicum FL27), M55228 [Rhizobium sp. (Medicago) OR191], Z95230 [Rhizobium sp. (Lonchocarpus) BR6001], J01781 (S. meliloti 41), M55232 (S. meliloti USDA $1002^{\mathrm{T}}$ ), M55232 (S. meliloti CC2013), M55231 (Sinorhizobium medicae CC169), Z95229 (S. fredii USDA 191), Z95218 (Sinorhizobium terangae bv. acaciae ORS $1009^{\mathrm{T}}$ ), SSZ95221 (Sinorhizobium saheli bv. sesbaniae ORS 609'), AE000105 (Sinorhizobium sp. NGR234), Z95224 [Sinorhizobium sp. (Acacia) HAMBI 1499], Z95212 [Sinorhizobium sp. (Leucaena) BR827], Z95213 [Sinorhizobium sp. (Prosopis) M6], Z95228 [Mesorhizobium sp. (Leucaena) INPA78B], K01620 (B. japonicum USDA 110), M16709 (Azorbizobium caulinodans ORS 571 ${ }^{\mathrm{T}}$ ), X51500 (Azospirillum brasilense Sp7), X03916 (Azotobacter chroococcum MCD1), X07866 (Rhodobacter capsulatus SB1003), J01740 (Klebsiella pneumoniae). The numbers for the $16 \mathrm{~S} \mathrm{rDNA}$ sequences were: U89831 (R. leguminosarum bv. viciae USDA 2508), X67227 (R. leguminosarum bv. trifolii ATCC 14480), U29388 (R. leguminosarum bv. phaseoli RCR3644), X67233 (R. tropici IIA CFN 299), X67234 (R. tropici IIB CIAT 899 $)$, U38469 ( $R$. tropici IIB CIAT 166), U28916 (R. etli CFN 42 ${ }^{\mathrm{T}}$ ), U28939 ( $R$. etli TAL182), U47303 (R. etli SEMIA0430), U86343 (R.

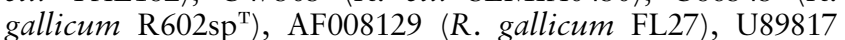
(Rhizobium mongolense USDA $1844^{\mathrm{T}}$ ), U89816 (R. mongolense USDA 1832), U89818 ( $R$. mongolense USDA 1834), U89819 ( $R$. mongolense USDA 1836), U89821 ( $R$. mongolense USDA 1890), U89823 [Rhizobium sp. (Medicago) USDA 1920], X91211 [Rhizobium sp. (Medicago) OR191], U29387 [Rhizobium sp. (Phaseolus) RCR3618D], U71078 (Rhizobium hainanense I66 $\left.{ }^{\mathrm{T}}\right), \mathrm{D} 12793$ ( $R$. galegae HAMBI $\left.540^{\mathrm{T}}\right)$, $\mathrm{AF} 025852\left(R\right.$. huautlense $\left.\mathrm{S} 02^{\mathrm{T}}\right)$, U86344 (R. giardinii $\left.\mathrm{H} 152^{\mathrm{T}}\right)$, D12783 (S. meliloti USDA $1002^{\mathrm{T}}$ ), D12783 (S. medicae A321 ${ }^{\mathrm{T}}$ ), X67231 (S. fredii USDA $205^{\mathrm{T}}$ ), X68388 (S. terangae ORS $1009^{\mathrm{T}}$ ), X68390 (S. sabeli ORS 609 ${ }^{\mathrm{T}}$ ), X67229 (M. loti NZP $2213^{\mathrm{T}}$ ), U50164 (M. loti R8CS), U50165 (M. loti R88b), U50166 (M. loti ICMP 3153), D12797 (Mesorhizobium huakuii CCBAU 2609 ${ }^{\mathrm{T}}$ ), U07934 (Mesorhizobium ciceri UPM$\mathrm{Ca}^{\mathrm{T}}$ ), L38825 (Mesorhizobium mediterraneum UPM-Ca36 ${ }^{\mathrm{T}}$ ), U71079 (Mesorhizobium tianshanense A-1BS ${ }^{\mathrm{T}}$ ), Y14158 (Mesorhizobium plurifarium LMG 11892 ${ }^{\mathrm{T}}$ ), AF041442 (Mesorhizobium amorphae ACCC $19665^{\mathrm{T}}$ ), U69638 (B. japonicum USDA $6^{\mathrm{T}}$ ), Z35330 (B. japonicum USDA 110), U35000 (B. elkanii USDA $76^{\mathrm{T}}$ ), X87273 [Bradyrhizobium sp. (Lupinus) DSM 30140], X70405 [Bradyrhizobium sp. (Acacia) LMG 10689], X70403 [Bradyrhizobium sp. (Acacia) LMG 9966], X70404 [Bradyrhizobium sp. (Enterolobium) LMG 9980], X70401 [Bradyrhizobium sp. (Lonchocarpus) LMG 9514], Y17047 (Allorbizobium undicola LMG 11875 ), X67221 (Azorhizobium caulinodans ORS 571 ${ }^{\mathrm{T}}$ ), X67223 (Agrobacterium tumefaciens LMG 196), X67228 (Agrobacterium rubi LMG 156 ${ }^{\mathrm{T}}$ ), X67225 (Agrobacterium vitis LMG 8750 ${ }^{\mathrm{T}}$ ), X67224 (Agrobacterium rhizogenes LMG 152).

Molecular sequence analyses were performed by using programs available in the Bisance software. Nucleotide and amino acid sequences were aligned with CLUSTAL w (Thompson et al., 1994). Phylogenetic trees of nodC, nifH and $16 \mathrm{~S}$ rRNA genes were inferred by using the Phylogenetic Inference Package (PHYLIP ; Felsenstein, 1989) with neighbourjoining analyses from Kimura's (Kimura, 1980) two-parameter nucleotide distances, and the maximum-likelihood method. Phylogenetic trees of NodC and NifH proteins were constructed using the neighbour-joining method from Dayhoff PAM distance matrix computed with the PROTDIST program of PHYLIP. Confidence in neighbour-joining trees was assessed by bootstrap analysis with the SEQBOOT and CONSENSE programs of PHYLIP.

\section{RESULTS AND DISCUSSION}

\section{PCR-amplification of nodC and nifH gene fragments}

The nodC gene fragment was amplified for the majority of the strains investigated $(67 / 82)$ by using primer pair nodCF-nodCI. Amplification was achieved for R. galegae HAMBI 540 by using either nodCF2 or nodCFu, and for B. elkanii USDA 76 by using nodCF4, instead of nodCF. Forward primer nodCFu was used with Bradyrhizobium sp. LB84 and WU425, and M. amorphae ACCC 19665. The nodC gene could not be amplified for the strains of the bv. sesbaniae of S. terangae and $S$. saheli by using the nodC primers described in this study, while PCR amplification was successful for the strains of $S$. saheli and S. terangae bv. acaciae. The bv. sesbaniae has been created recently to group $S$. saheli and $S$. terangae strains isolated from Sesbania spp. that showed similar genetic and phenotypic symbiotic features (Lortet et al., 1996; Haukka et al., 1998). The absence of an amplification product was probably due to some nucleotide mismatches in nodC genes of bv. sesbaniae strains with the $3^{\prime}$ end region of one or both oligonucleotides used as nodC primer pairs. Amplification of the nifH fragment was achieved for all the 76 strains investigated, except for $R$. leguminosarum bv. trifolii cc2480a for unknown reasons, and for $R$. giardinii bv. giardinii $\mathrm{H} 152$ as expected since strains in this biovar probably lack nifKDH genes (Geniaux et al., 1993; Amarger et al., 1997).

\section{Correlation between RFLP of Sym genes and host specificity}

The results of the RFLP analysis of the PCR-amplified nod $\mathrm{C}$ and nifH fragments are given in Table 1 . From the combined data with each gene, we identified 45 composite nod types and 41 nif types among the 82 and 76 strains investigated, respectively. The combination of 
the nod and nif gene analyses revealed 50 symbiotic (nod-nif) genotypes. We concluded that both genes were highly polymorphic among species and biovars.

Intraspecies polymorphism was also detected among strains isolated from the same host legume (in R. tropici, S. meliloti, M. loti and M. huakuii) and, irrespective of the species, within previously defined biovars (viciae, trifolii, phaseoli, gallicum, sesbaniae), but differences between pairs of restriction patterns could be simply explained in terms of gain or loss of only one or two restriction sites. These results indicate that the genes are closely related.

Unclassified strains could be assigned to previously defined biovars. Thus Rhizobium sp. (Phaseolus) RCR3618D had Sym genes characteristic of bv. phaseoli. Rhizobium sp. (Leucaena) USDA 3497 could be classified into bv. gallicum, which is consistent with the ability of $R$. gallicum bv. gallicum to nodulate Leucaena leucocephala (Amarger et al., 1997).

More generally and independent of their taxonomic status, the strains sharing at least two similar nodC and/or nifH restriction patterns originated from host plants belonging to the same species or genus, or to the same known cross-inoculation group. The Rhizobium sp. (Phaseolus) strains HT2a2 and HT4c1 had nodC genes typical of $R$. tropici. The soybean bradyrhizobia $B$. japonicum and Bradyrhizobium liaoningense had identical nod and nif types. Most restriction patterns were similar in the chickpea mesorhizobia M. ciceri and $M$. mediterraneum. The strains of $R$. mongolense and Rhizobium sp. OR191 which originated from Medicago species shared four nodC restriction patterns and the ability to form nitrogen-fixing nodules with common beans (Eardly et al., 1992; van Berkum et al., 1998). However, only the latter was reported to nodulate Leucaena leucocephala (Del Papa et al., 1999). The Medicago sinorhizobia S. meliloti and S. medicae also showed two identical nodC restriction patterns, which indicates that they have closely related nodC genes. Similar nifH restriction patterns were obtained among the $R$. huautlense strains isolated from Sesbania herbacea and the bv. sesbaniae of $S$. terangae and $S$. saheli, showing that at least their nifH genes are closely related. However, the nodC genes of the $R$. buautlense strains were easily amplified by contrast to those of the Sesbania sinorhizobia. This result indicated some nucleotide differences between their nodC genes but further investigations are needed to estimate to what extent their nodulation genes differ. The five unclassified bradyrhizobia isolated from Lupinus or Ornithopus species, two legume genera that form a single crossinoculation group (Graham, 1976), also had closely related nifH genes.

Conversely, strains with no known common host plants shared few restriction patterns (and no more than one in pairwise comparisons). However, there were also strains among Phaseolus, Medicago, Acacia or soybean rhizobia that did not share more than one restriction pattern. The RFLP method was not suitable for ob- taining more information about phylogenetic relationships between the genes. Few restriction sites in nodC and nif $H$ gene sequences were actually conserved among species or biovars, according to the available nucleotide sequences. Therefore, it was not possible to map the restriction sites for rigorous phylogenetic analyses, and so further nucleotide sequencing was required.

\section{Relationship of phylogeny of Sym genes to host specificity}

Complete or partial sequences of the PCR-amplified nodC fragments were determined for representatives of the different RFLP nod-nif types among strains that originated from Phaseolus vulgaris nodules, and for $R$. leguminosarum bv. trifolii M37, Rhizobium sp. OR191 from Medicago sativa, and the type strain ACCC 19665 of the recently described species $M$. amorphae (Wang et al., 1999b). We also determined the sequence of the nif $H$ fragment of R. gallicum bv. gallicum R602sp and of the Sinorhizobium sp. (Phaseolus) strains GR-06 and GRX8. Restriction site mapping and comparisons with the experimental RFLP data confirmed the quality of the sequences. Phylogenetic analysis of nodC and nifH sequences was performed by using the neighbour-joining and the maximum-likelihood methods, which led to similar results, except for some uncertain nodes which were not supported by high bootstrap values ( $>85 \%$ over 500 replicates) in the nifH neighbour-joining tree.

The $\operatorname{nod} C$ trees were also similar to that derived from protein translation of the DNA sequences and only the neighbour-joining tree is shown in Fig. 2. The nodC phylogeny was well correlated with the host plant range. All the nodC genes but one of the Phaseolus rhizobia formed a robust cluster within which the similarity values ranged from 81.9 to $99.5 \%$. This result suggests that these nod genes evolved from a common ancestor. $R$. tropici fell outside the bean symbiont cluster and the present data did not show evidence that $R$. tropici was more strongly associated with the bean symbionts than with other rhizobia. Each of the bvs phaseoli, gallicum and giardinii corresponded to distinct lineages or subclusters, which correlates with the differences observed in host plant range between these biovars (Amarger et al., 1997; Sessitsch et al., 1997). However, a close relationship was found between the nodC genes of $R$. gallicum bv. gallicum and R. giardinii bv. giardinii, a result that could not have been anticipated from the RFLP analysis. The similarity values were higher than $90 \%$. Strain GR-06 formed its own lineage within the bean symbiont cluster, and consequently this strain could not be assigned to any of the previously defined biovars.

The nodC gene of Rhizobium sp. (Medicago) OR191 clustered with the $S$. meliloti nodC gene, its closest relative $(87.8 \%$ homology), suggesting that these genes originated from a common ancestor. OR191 was reported to form ineffective nodules with its host of origin, Medicago sativa, but to be symbiotically more effective with common bean (Eardly et al., 1992). 


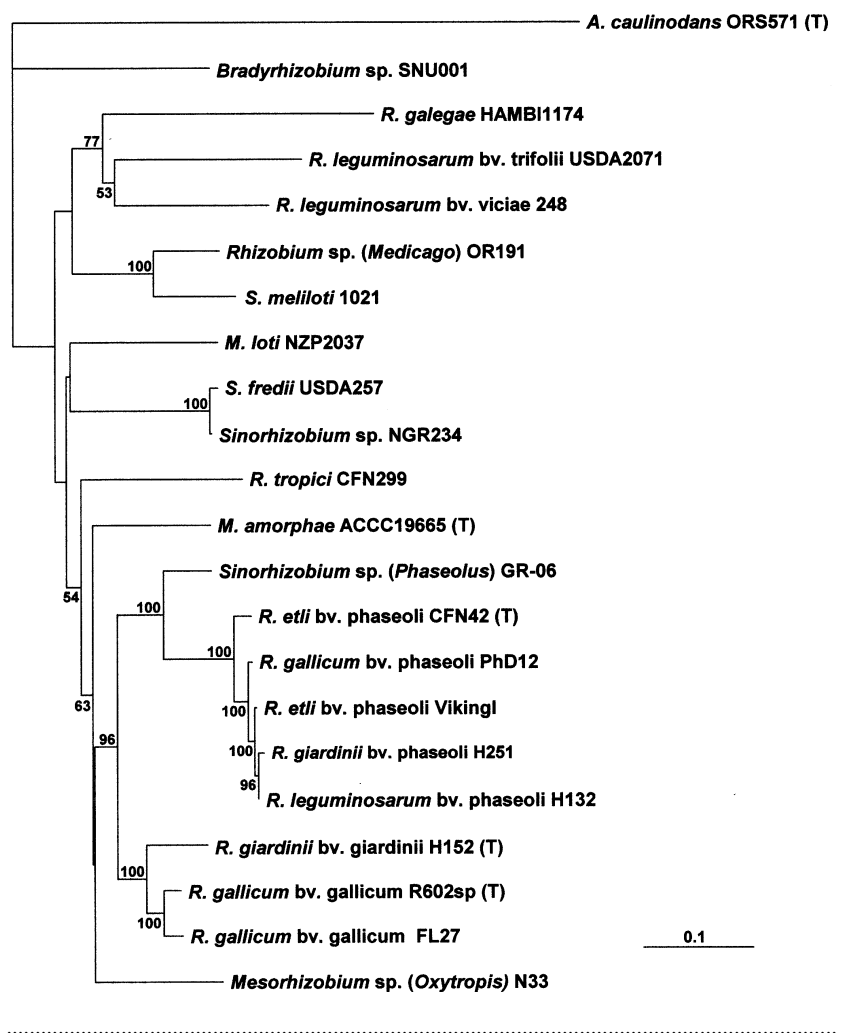

Fig. 2. Phylogenetic (neighbour-joining) tree based on $781 \mathrm{bp}$ aligned sequences of nodC (positions 337-1113 of the nucleotide sequence of the $R$. leguminosarum bv. viciae nodC gene). Only bootstrap probability values greater than $50 \%$ (over 100 replicates) are indicated at the branching points. The horizontal branches are drawn proportionally to the number of nucleotide substitutions per site. A., Azorhizobium; B., Bradyrhizobium; M., Mesorhizobium; R., Rhizobium; S., Sinorhizobium; $(T)$, type strain.

However, phylogenetic analysis clearly showed that the nodC gene of OR191 was relatively distant from those of the Phaseolus symbionts.

The nifH neighbour-joining tree is shown in Fig. 3. A tree derived from the protein translation was also constructed (not shown), but the phylogenetic analysis was not reliable since the bootstrap values were generally low $(<50 \%)$. Analysis of longer nucleotide sequences of nifH (738 bp aligned) improved the general robustness of nifH phylogenetic trees (not shown), but the number of rhizobial sequences available for comparison was too small for phylogenetic use. Our neighbour-joining tree was similar to those reported previously (Haukka et al., 1998; Wang et al., 1999a). As in the nodC tree, R. gallicum bv. gallicum formed a tight cluster with R. etli bv. phaseoli, which also included $R$. etli bv. mimosae Mim2. This strain was isolated from Mimosa affinis but is able to nodulate $P$. vulgaris and Leucaena leucocephala like strains of $R$. gallicum bv. gallicum (Wang et al., 1999a). On the basis of the lack of polymorphism revealed by the RFLP analysis (Table 1), it seems probable that the nifH genes are very similar among all the strains classified in bv. phaseoli, whatever

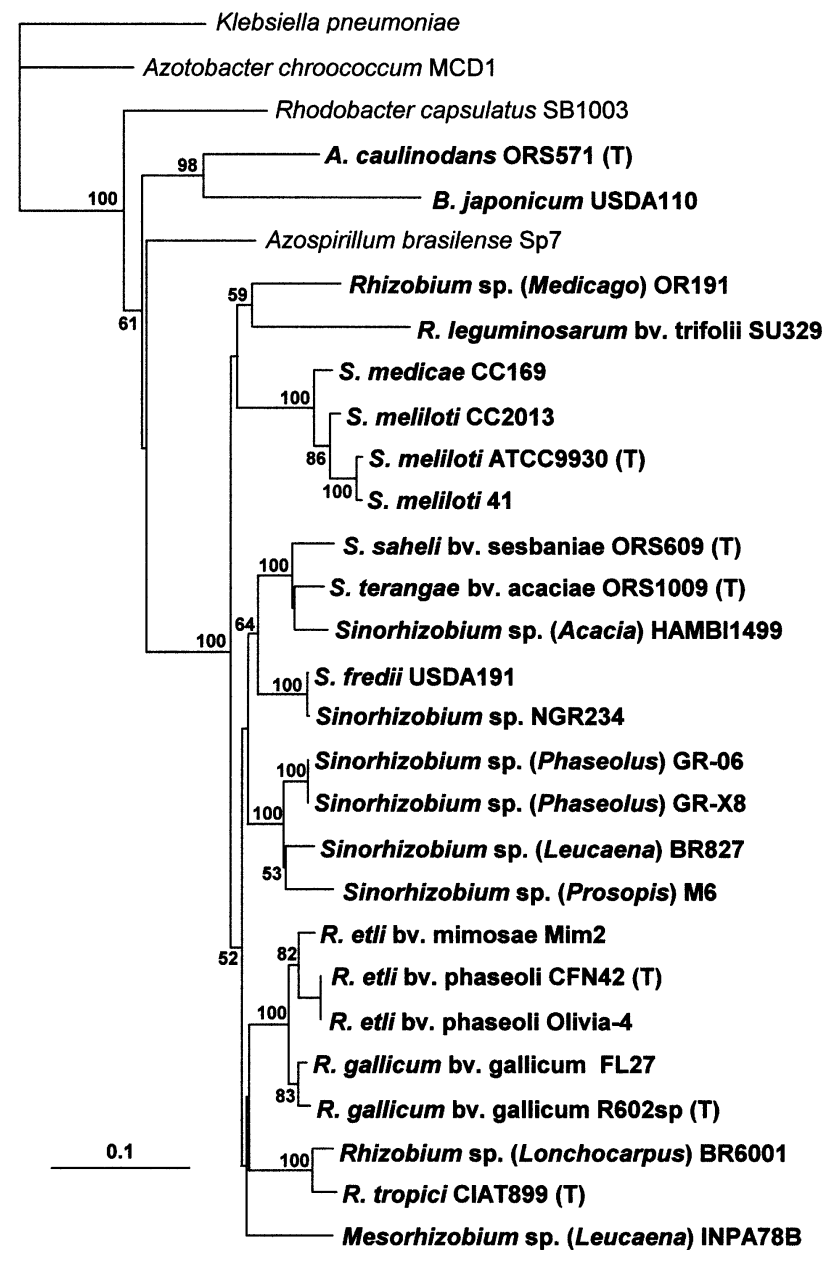

Fig. 3. Phylogenetic (neighbour-joining) tree based on $475 \mathrm{bp}$ aligned sequences of nifH (positions 312-786 of the nucleotide sequence of the $R$. leguminosarum bv. trifolii nifH gene). Only bootstrap probability values greater than $50 \%$ (over 500 replicates) are indicated at the branching points. The horizontal branches are drawn proportionally to the number of nucleotide substitutions per site. A., Azorhizobium; B., Bradyrhizobium; M., Mesorhizobium; R., Rhizobium; S., Sinorhizobium; (T), type strain.

the species. By contrast, but consistent with the RFLP data in $R$. gallicum bv. gallicum, the nifH nucleotide sequence of R602sp, the type strain of the species isolated in France, differed from that of the Mexican strain FL27 at $2 \%$ of nucleotide sites. This intra-biovar difference was relatively high compared to the differences observed between R602sp and R. etli bvs mimosae and phaseoli strains, which were only of 3.3 and $3.5 \%$, respectively. The grouping of the two R. gallicum bv. gallicum strains into a subcluster was supported by an $83 \%$ bootstrap value, but not corroborated by the maximum-likelihood tree, in which the two R. gallicum strains and R. etli bv. mimosae Mim2 each formed a distinct lineage within the Phaseolus cluster. So far, almost all isolates of $R$. gallicum have been obtained from Europe (Amarger et al., 1997; Sessitsch et al., 1997; Herrera-Cervera et al., 1999). Because of the Mesoamerican origin of bean and 
of R. gallicum strain FL27, Sessitsch et al. (1997) suggested that $R$. gallicum might have been imported to Europe as a seed contaminant. However, taking into account the relative divergence among the Sym genes of the French and Mexican R. gallicum bv. gallicum strains, this hypothesis appears unlikely.

The $R$. tropici sequence was positioned inside a clade including the R. etli-R. gallicum cluster (Fig. 3). The result was supported by the maximum-likelihood analysis, but not corroborated by the bootstrap analysis. The Phaseolus sinorhizobia GR-06 and GR-X8 have similar nifH gene sequences which showed a significant close relationship only with two tropical sinorhizobia isolated from Leucaena and Prosopis spp. trees (Haukka et al., 1998). The relatedness of this cluster to the $R$. gallicum-R. etli cluster could not be resolved. In the neighbour-joining tree, the cluster including the Phaseolus sinorhizobia fell outside the R. gallicum $-R$. etli cluster, while in the maximum-likelihood tree, the two clusters grouped in a clade. However, the $\operatorname{nod} A$ genes of the tree sinorhizobia BR827 and M6 did not appear to be closely related to that of R. etli (Haukka et al., 1998). Although we lack extensive data to assume that $\operatorname{nod} A$ and $\operatorname{nod} C$ phylogenies are equivalent, this seems likely based on previous phylogenetic surveys (Lindström et al., 1995; Ueda et al., 1995; Zhang et al., 2000). No case of incongruence was detected by comparing our nodC tree with the nodA tree from Haukka et al. (1998), and congruence between nodB and nodC phylogenies, at least within the Rhizobium and Sinorhizobium genera, was also reported by Wernegreen $\&$ Riley (1999). If so, that would mean that nod genes of GR-06 are not closely related to those of the sinorhizobia BR827 and M6 and, consequently, that nod and nif genes did not co-evolve together in GR-06-like strains. The nod genes may be under a higher selective pressure to adapt to host differences, or alternatively, the nod and nif genes were not acquired together.

The second major difference between the nodC and nifH trees concerned the Medicago strain OR191, which was not associated with the tight cluster formed by $S$. melilot $i$ and $S$. medicae in the nif $H$ trees. The nif $H$ sequence of OR191 formed its own lineage. Our updated analysis confirmed the earlier results of Eardly et al. (1992) indicating that this sequence was substantially different from those of the other rhizobia (similarity values lower than $89 \%$ ). Again, the data suggest that the nodC and the nifH genes did not co-evolve together in this strain. The nodC gene of OR191 could be the ancestor from which Medicago strain genes diverged. Alternatively, OR191, and also GR-06-like strains, may have acquired only the nod genes by horizontal transfer from hostspecific ancestral nod genes. Since nod and nif genes are often closely linked, another hypothesis would be that additional events of gene exchange and internal genetic rearrangements might have followed the co-transfer of the nod and nif genes. High-frequency rearrangements in plasmids of rhizobial strains involving recombination among reiterated sequences have been reported (see Garcia-de los Santos et al., 1996; Romero et al., 1998).
Previous reports also indicated that some rhizobial strains, such as $S$. saheli, had different phylogenetic locations within rhizobia by comparing common nod gene and nifH evolutionary trees (Dobert et al., 1994; Haukka et al., 1998). Clearly, more work is needed to assess the linkage of nod and nif genes and the rest of the symbiotic genome.

\section{Incongruence between symbiotic types and 16S-rDNA-based classification}

Our results extend previously reported evidence showing that distinct rhizobial species can share similar Sym genes, and, conversely, that distinct Sym genotypes and phenotypes can be harboured by similar genomic backgrounds as defined by $16 \mathrm{~S}$ rDNA types. Also, we observed additional cases of incongruence between the classifications and phylogenies resulting from comparative analyses of Sym genes and 16S rRNA (Figs 1-3).

The 16S rDNA type of Rhizobium sp. bv. gallicum USDA 3497 matched that of the $R$. mongolense type strain (Table 1), though it should be noted that this type is closely related to that of R. gallicum (Laguerre et al., 1997; van Berkum et al., 1998; Fig. 1). On the other hand, the nodC gene of $R$. mongolense was found to be close to that of the Medicago rhizobium OR191 (Table 1) and then probably distant from those of bv. gallicum on the basis of sequence analysis of the OR191 nodC gene. Although R. mongolense strains formed nitrogenfixing nodules on P. vulgaris (van Berkum et al., 1998), they were not able to nodulate Leucaena leucocephala in contrast with strains in bv. gallicum.

Strains HT2a2 and HT4c1 harboured nodC genes typical of $R$. tropici, but their $16 \mathrm{~S} \mathrm{rDNA}$ type was identical to that of strain OR191 (Table 1). This $16 \mathrm{~S}$ rDNA type differed in nine restriction sites from that of the R. tropici type strain (Laguerre et al., 1997), and the sequence data indicated that strain OR191 does not show significant phylogenetic affinity for $R$. tropici or any other rhizobial lineage (Fig. 1). Strains OR191, HT2a 2 and HT4c1 may form a new Rhizobium species that would harbour different Sym genes.

The classification resulting from the Sym gene analysis fully reflected the host specificity for the sample of mesorhizobia investigated, while the 16S-rDNA-based phylogeny was irrespective of the host plant (Sullivan et al., 1996; Laguerre et al., 1997; de Lajudie et al., 1998b; Fig. 1). In particular, the sample of four strains of $M$. loti examined represented three distinct $16 \mathrm{~S}$ rDNA types intermixed with other mesorhizobia from various host legumes (Laguerre et al., 1994, 1997), but they had clearly closely related nodC and nif $H$ genes.

Similarly, the samples of soybean and lupin bradyrhizobial strains investigated in this study constituted heterogeneous 16S rDNA groups that were phylogenetically intermixed with each other and with bradyrhizobia isolated from other host legumes (Laguerre et al., 1994, 1997). The nodC gene was polymorphic among the lupin bradyrhizobia, but the nif $H$ gene sequences appear to be conserved within this group (Table 1). The 
soybean species B. japonicum and B. liaoningense that were delineated by $16 \mathrm{~S} \mathrm{rDNA}$ sequence comparison $(\mathrm{Xu}$ et al., 1995) harboured similar Sym genes. The B. elkanii strains had a specific Sym RFLP type, but former phylogenetic analyses had established that the common nodulation genes of B. japonicum and B. elkanii were closely related (Dobert et al., 1994; Ueda et al., 1995). However, further studies are necessary to investigate whether the Sym gene phylogeny is correlated with the $16 \mathrm{~S}$ rRNA classification and host range within the Bradyrhizobium genus, which includes a wide variety of yet unclassified microsymbionts associated with many legumes.

The nod genes of the soybean symbionts $S$. fredii and $B$. japonicum have been reported as being of the same lineage (Dobert et al., 1994), but the result was not supported by bootstrap analysis, as confirmed by more recent phylogenetic surveys inferred from nodA (Haukka et al., 1998) and nodC (Prévost et al., 2000) genes. Also, the nifH genes were not found to be related in these two species (Dobert et al., 1994; Haukka et al., 1998; Fig. 3). Therefore, among the soybean symbionts, the Sym gene phylogenies agree with the 16S rRNA phylogeny at the genus level.

By contrast, the nodC phylogeny was not only irrespective of the classification into species but also of the classification into genera among the Phaseolus rhizobia. Indeed, the highest similarity values $(99-99.5 \%$ ) between the nodC genes of the Phaseolus rhizobia were found between species rather than within species (only $96.5 \%$ similarity between the two R. etli bv. phaseoli strains, and a maximum of $84.6 \%$ between biovars within $R$. gallicum and $R$. giardinii). Almost all the Phaseolus symbionts belong to the genus Rhizobium, but GR-06-like strains were classified into the genus Sinorbizobium. The $16 \mathrm{~S}$ rDNA sequence of strain GR06 was found to be identical to that of $S$. fredii (HerreraCervera et al., 1999), but their Sym genes are not closely related (Figs 2 and 3). These results are consistent with the observation that GR-06-like strains were not able to nodulate soybean (Herrera-Cervera et al., 1999). R. giardinii provides further evidence of incongruence between nodC and 16S-rDNA-based phylogenies, the latter indicating that $R$. giardinii would deserve a genus status distinct from the genera described so far (Amarger et al., 1997), as confirmed by our updated phylogenetic tree (Fig. 1).

Additional evidence of discrepancy between Sym gene phylogeny and 16S-rDNA-based classification of rhizobia in genera was obtained within the Medicago and the Sesbania symbionts (Table 1; Figs 1 and 2). A similar case was reported by Wernegreen \& Riley (1999) for Rhizobium sp. strains isolated from Glycyrrhiza and Hedysarum species that had nod genes closely related to those of Medicago sinorhizobia.

\section{Specificity of the rhizobia-P. vulgaris associations}

The Phaseolus rhizobia investigated in this study constitute a representative sample of the Phaseolus symbionts described so far. For all the strains except R. tropici, the ability to establish a symbiosis in beans appears to be directed by a specific set of diversified but closely related nodulation genes. This should lead to a reconsideration of the usually accepted view that $P$. vulgaris is an undiscriminating host based on the diversity of its microsymbionts and the fact that most rhizobia studied so far are able to nodulate $P$. vulgaris when tested in laboratory assays (Martinez et al., 1985; Michiels et al., 1998). Moulin et al. (2000) indicated that nodA phylogeny gives indications on structural features of Nod factors. It is possible that the bean symbionts that have closely related nod genes produce similar Nod factors adapted for specific bean receptors. These Sym genes may confer upon the bacteria that harbour them a good competitive ability for nodule formation on beans. Furthermore, bean isolates that were effective in nitrogen fixation have been found in each species and biovar in which they are included (Young, 1985; Martinez-Romero et al., 1991; Segovia et al., 1993; Amarger et al., 1994, 1997; Wang et al., 1999a), except in R. giardinii (Amarger et al., 1997). By contrast, the other rhizobia capable of nodulating bean plants as single-strain inoculants generally produce ineffective nodules (Martinez et al., 1985; Michiels et al., 1998). Taken together, these data suggest a certain specificity in the rhizobia-bean symbiosis.

\section{Gene transfer is probably involved in evolution of the symbiotic functions}

Assuming that R. etli bv. phaseoli strains would be the symbionts that co-evolved with $P$. vulgaris, it has been hypothesized that they may be the original donors of the Sym plasmid in R. leguminosarum bv. phaseoli (Segovia et al., 1993). Likewise, R. gallicum and R. giardinii bv. phaseoli probably received their Sym plasmids from $R$. etli bv. phaseoli or, more plausibly, from the $R$. leguminosarum bv. phaseoli strains which co-existed in the fields in which the $R$. gallicum and $R$. giardinii isolates originated (Amarger et al., 1997). The hypothesis of interspecies gene transfer is supported by the high similarity of the Sym genes among the bv. phaseoli subgroups and the co-occurrence of all these species in Europe (Geniaux et al., 1993; Sessitsch et al., 1997; Herrera-Cervera et al., 1999). In the same way, the finding that the Sym genes of strains HT2a2 and HT4c1 and of $R$. tropici are similar although their $16 \mathrm{~S}$ rDNA types are relatively distant, and the co-occurrence of these rhizobia in the southwest of France (Amarger et al., 1994), argue for interspecies gene transfer. Hence, these data support the view that gene transfer would play a role in diversification and in structuring the natural populations of rhizobia, notably those nodulating P. vulgaris.

Furthermore, this work has also revealed the probable common origin of nod and nif genes among rhizobia belonging to the Rhizobium and Sinorhizobium genera that nodulate Phaseolus, Medicago and Sesbania. These findings suggest gene exchange events across genera, 
which does not support the view of Wernegreen \& Riley (1999) that the Sym genes in rhizobial genera diverge independently. Additionally, analysis of glutamine synthetase genes also suggests transfers of genes between the rhizobial genera (Turner \& Young, 2000).

The comparison of the nod, nif and 16S rRNA phylogenies and the substantial correlation that we found between symbiotic genotypes and host plant groups taken together support the generally accepted hypothesis that lateral transfer of Sym genes and genetic rearrangements are involved in the acquisition and evolution of rhizobial symbiotic functions.

\section{ACKNOWLEDGEMENTS}

We thank M. R. Allard, M. Bours, F. Maillard, F. Revoy, S. Munoz and J. A. Herrera-Cervera for technical assistance. We are very grateful to all the scientists who sent us strains, and also to the referees who significantly contributed to improving this manuscript.

This work was funded by INRA as part of AIP Microbiologie, and by MESR as part of ACC-SV no. 6. It was also financially supported by a grant from the Conseil Régional de Bourgogne.

\section{REFERENCES}

Amarger, N., Bours, M., Revoy, F., Allard, M. R. \& Laguerre, G. (1994). Rhizobium tropici nodulates field-grown Phaseolus vulgaris in France. Plant Soil 161, 147-156.

Amarger, N., Macheret, V. \& Laguerre, G. (1997). Rhizobium gallicum sp. nov. and R hizobium giardinii sp. nov. from Phaseolus vulgaris nodules. Int J Syst Bacteriol 47, 996-1006.

Bergersen, F. J., Turner, G. L., Amarger, N., Mariotti, N. \& Mariotti, A. (1986). Strain of Rhizobium lupini determines natural abundance of $15 \mathrm{~N}$ in root nodules of Lupinus spp. Soil Biol Biochem 18, 97-101.

van Berkum, P., Beyene, D. \& Eardly, B. D. (1996). Phylogenetic relationships among Rhizobium species nodulating the common bean (Phaseolus vulgaris L.). Int J Syst Bacteriol 46, 240-244.

van Berkum, P., Beyene, D., Bao, G., Campbell, T. A. \& Eardly, B. D. (1998). Rhizobium mongolense sp. nov. is one of three rhizobial genotypes identified which nodulate and form nitrogen-fixing symbioses with Medicago ruthenica [(L.) Ledebour]. Int J Syst Bacteriol 48, 13-22.

Chen, W., Wang, E., Wang, S., Li, Y., Chen, X. \& Li, Y. (1995). Characteristics of Rhizobium tianshanense sp. nov., a moderately and slowly growing root nodule bacterium isolated from an arid saline environment in Xianjiang, People's Republic of China. Int J Syst Bacteriol 45, 153-159.

Del Papa, M. F., Balagué, L. J., Castro Sowinski, S. \& 9 other authors (1999). Isolation and characterization of alfalfa-nodulating rhizobia present in acidic soils of central Argentina and Uruguay. Appl Environ Microbiol 65, 1420-1427.

Dessen, P., Fondrat, C., Valencien, C. \& Mugnier, C. (1990). Bisance: a French service for access to biomolecular sequence databases. Comput Appl Biosci 6, 355-356.

Dobert, R. C., Breil, B. T. \& Triplett, E. W. (1994). DNA sequence of the common nodulation genes of Bradyrhizobium elkanii and their phylogenetic relationships to those of other nodulating bacteria. Mol Plant-Microbe Interact 7, 564-572.

Eardly, B. D., Young, J. P. W. \& Selander, R. K. (1992). Phylogenetic position of Rhizobium sp. strain Or 191, a symbiont of both Medicago sativa and Phaseolus vulgaris, based on partial sequences of the 16S rRNA and nifH genes. Appl Environ Microbiol 58, 1809-1815.

Felsenstein, J. (1989). PHYLIP - phylogeny inference package (version 3.2). Cladistics 5, 164-166.

Garcia-de los Santos, A., Brom, S. \& Romero, D. (1996). Rhizobium plasmids in bacteria-legume interactions. World J Microbiol Biotechnol 12, 119-125.

Geniaux, E., Laguerre, G. \& Amarger, N. (1993). Comparison of geographically distant populations of Rhizobium isolated from root nodules of Phaseolus vulgaris. Mol Ecol 2, 295-302.

Graham, P. H. (1976). Identification and classification of root nodule bacteria. In Symbiotic Nitrogen Fixation in Plants, pp. 99-112. Edited by P. S. Nutman. International Biological Programme No. 7. London: Cambridge University Press.

Graham, P. H., Sadowsky, M. J., Keyser, H. H. \& 8 other authors (1991). Proposed minimal standards for the description of new genera and species of root- and stem-nodulating bacteria. Int $J$ Syst Bacteriol 41, 582-587.

Haukka, K., Lindström, K. \& Young, J.P. W. (1998). Three phylogenetic groups of nodA and nifH genes in Sinorhizobium and Mesorbizobium isolated from leguminous trees growing in Africa and Latin America. Appl Environ Microbiol 64, 419-426.

Hennecke, H., Kaluza, K., Thöny, B., Fuhrmann, M., Ludwig, W. \& Stackebrandt, E. (1985). Concurrent evolution of nitrogenase genes and 16S rRNA in Rhizobium species and other nitrogen fixing bacteria. Arch Microbiol 142, 342-348.

Herrera-Cervera, J. A., Caballero-Medallo, J., Laguerre, G., Tichy, H. V., Requena, N., Amarger, N., Martinez-Romero, E., Olivares, J. \& Sanjuan, J. (1999). At least five rhizobial species nodulate Phaseolus vulgaris in a Spanish soil. FEMS Microbiol Ecol 30, 87-97.

Kimura, M. (1980). A simple method for estimating evolutionary rates of base substitutions through comparative studies of nucleotide sequences. J Mol Evol 16, 111-120.

Kuykendall, L. D., Saxena, B., Devine, T. E. \& Udell, S. E. (1992). Genetic diversity in Bradyrhizobium japonicum Jordan 1982 and a proposal for Bradyrhizobium elkanii sp. nov. Can J Microbiol 38, 501-505.

Lagacherie, B., Hugot, R. \& Amarger, N. (1977). Sélection de souches de Rhizobium japonicum d'après leur compétitivité pour l'infection. Ann Agron 28, 379-389.

Lagacherie, B., Bours, M., Giraud, J. J. \& Sommer, G. (1983). Interactions entre différentes souches de Rhizobium lupini et les espèces ou cultivars de lupin (Lupinus albus, Lupinus luteus et Lupinus mutabilis). Agronomie 3, 809-816.

Laguerre, G., Allard, M. R., Revoy, F. \& Amarger, N. (1994). Rapid identification of rhizobia by restriction fragment length polymorphism analysis of PCR-amplified $16 \mathrm{~S}$ rRNA genes. Appl Environ Microbiol 60, 56-63.

Laguerre, G., Mavingui, P., Allard, M. R., Charnay, M. P., Louvrier, P., Mazurier, S. I., Rigottier-Gois, L. \& Amarger, N. (1996). Typing of rhizobia by PCR DNA fingerprinting and PCR-restriction fragment length polymorphism analysis of chromosomal and symbiotic gene regions: application to Rhizobium leguminosarum and its different biovars. Appl Environ Microbiol 62, 2029-2036.

Laguerre, G., van Berkum, P., Amarger, N. \& Prévost, D. (1997). Genetic diversity of rhizobial symbionts isolated from legume species within the genera Astragalus, Oxytropis and Onobrychis. Appl Environ Microbiol 63, 4748-4758.

de Lajudie, P., Willems, A., Pot, B. \& 7 other authors (1994). Polyphasic taxonomy of rhizobia: emendation of the genus 
Sinorhizobium, and description of Sinorhizobium meliloti comb. nov., Sinorhizobium sabeli sp. nov., and Sinorhizobium teranga sp. nov. Int J Syst Bacteriol 44, 715-733.

de Lajudie, P., Laurent-Fulele, E., Willems, A., Torck, U., Coopman, R., Collins, M. D., Kersters, K., Dreyfus, B. \& Gillis, M. (1998a). Allorbizobium undicola gen. nov., sp. nov., nitrogen-fixing bacteria that efficiently nodulate Neptunia natans in Senegal. Int J Syst Bacteriol 48, 1277-1290.

de Lajudie, P., Willems, A., Nick, G. \& 9 other authors (1998b). Characterization of tropical tree rhizobia and description of Mesorhizobium plurifarium sp. nov. Int J Syst Bacteriol 48, 369-382.

Lindström, K. (1989). Rhizobium galegae, a new species of legume root nodule bacteria. Int J Syst Bacteriol 39, 365-367.

Lindström, K., Paulin, L., Roos, C. \& Suominen, L. (1995). Nodulation genes of Rhizobium galegae. In Nitrogen Fixation: Fundamentals and Applications. Proceedings of the 10th International Congress on Nitrogen Fixation, pp. 365-370. Edited by I. A. Tikhonovitch, N. A. Provorov, V. I. Romanov \& W. E. Nexton. Dordrecht: Kluwer.

Lortet, G., Méar, N., Lorquin, J., Dreyfus, B., de Lajudie, P., Rosenberg, C. \& Boivin, C. (1996). Nod factor thin-layer chromatography profiling as a tool to characterize symbiotic specificity of rhizobial strains: application to Sinorhizobium sabeli, S. teranga, and Rhizobium sp. strains isolated from Acacia and Sesbania. Mol Plant-Microbe Interact 9, 736-747.

Martinez, E., Pardo, M. A., Palacios, R. \& Cevallos, M. A. (1985). Reiteration of nitrogen fixation gene sequences and specificity to Rhizobium in nodulation and nitrogen fixation in Phaseolus vulgaris. J Gen Microbiol 131, 1779-1786.

Martinez-Romero, E. \& Caballero-Mellado, J. (1996). Rhizobium phylogenies and bacterial genetic diversity. Crit Rev Plant Sci 15, 113-140.

Martinez-Romero, E., Segovia, L., Mercante, F. M., Franco, A. A., Graham, P. \& Pardo, M. A. (1991). Rhizobium tropici, a novel species nodulating Phaseolus vulgaris $\mathrm{L}$. beans and Leucaena sp. trees. Int J Syst Bacteriol 41, 417-426.

Michiels, J., Dombrecht, B., Vermeiren, N., Xi, C., Luyten, E. \& Venderleyden, J. (1998). Phaseolus vulgaris is a non-selective host for nodulation. FEMS Microbiol Ecol 26, 193-205.

Moulin, L., Debellé, F., Giraud, E., Mangin, B., Dénarié, J. \& Boivin-Masson, C. (2000). The nodA sequence of rhizobia gives clues on structural features of Nod factors. In Nitrogen Fixation: From Molecules to Crop Productivity, p. 204. Edited by F. O. Pedrosa, M. Hungria, M. G. Yates \& W. E. Newton. Dordrecht: Kluwer.

Perret, X., Staehelin, C. \& Broughton, W. J. (2000). Molecular basis of symbiotic promiscuity. Microbiol Mol Biol Rev 64, 180-201.

Poupot, R., Martinez-Romero, E. \& Promé, J. C. (1993). Nodulation factors from Rhizobium tropici are sulfated or nonsulfated chitopentasaccharides containing an $\mathrm{N}$-methyl- $\mathrm{N}$-acylglucosamine terminus. Biochemistry 32, 10430-10435.

Poupot, R., Martinez-Romero, E., Gautier, N. \& Promé, J. C. (1995). Wild type Rhizobium etli, a bean symbiont, produces acetyl-fucosylated, $\mathrm{N}$-methylated, and carbamoylated nodulation factors. J Biol Chem 270, 6050-6055.

Prévost, D., Macheret, V. \& Laguerre, G. (2000). Phylogenetic comparisons of symbiotic (nodC and nifH) and 16S rDNA genes in strains of Rhizobium, Mesorhizobium and Bradyrhizobium isolated from Astragalus, Oxytropis and Onobrychis spp. In Nitrogen Fixation: From Molecules to Crop Productivity, p. 205.
Edited by F. O. Pedrosa, M. Hungria, M. G. Yates \& W. E. Newton. Dordrecht: Kluwer.

Pueppke, S. G. \& Broughton, W. J. (1999). Rhizobium sp. strain NGR234 and R. fredii USDA257 share exceptionally broad, nested host ranges. Mol Plant-Microbe Interact 12, 293-318.

Romero, D., Davila, G. \& Palacios, R. (1998). The dynamic genome of Rhizobium. In Bacterial Genomes: Physical Structure and Analysis, pp. 153-161. Edited by F. J. de Bruijn, J. R. Lupski \& G. M. Weinstock. New York: Chapman \& Hall, International Thompson Publishing.

Segovia, L., Young, J. P. W. \& Martinez-Romero, E. (1993). Reclassification of American Rhizobium leguminosarum biovar phaseoli type I strains as Rhizobium etli sp. nov. Int J Syst Bacteriol 43, 374-377.

Sessitsch, A., Ramirez-Saad, H., Hardarson, G., Akkermans, A. D. I. \& de Vos, W. M. (1997). Classification of Austrian rhizobia and the Mexican isolate FL27 obtained from Phasolus vulgaris L. as Rhizobium gallicum. Int J Syst Bacteriol 47, 1097-1101.

Sullivan, J. T. \& Ronson, C. W. (1998). Evolution of rhizobia by acquisition of a $500-\mathrm{kb}$ symbiosis island that integrates into a phetRNA gene. Proc Natl Acad Sci US A 95, 5145-5149.

Sullivan, J. T., Patrick, H. N., Lowther, W. L., Scott, D. B. \& Ronson, C. W. (1995). Nodulating strains of Rhizobium loti arise through chromosomal symbiotic gene transfer in the environment. Proc Natl Acad Sci US A 92, 8985-8989.

Sullivan, J. T., Eardly, B. D., van Berkum, P. \& Ronson, C. W. (1996). Four unnamed species of nonsymbiotic rhizobia isolated from the rhizosphere of Lotus corniculatus. Appl Environ Microbiol 62, 2818-2825.

Thompson, J. D., Higgins, C. W. \& Gibson, T. J. (1994). CLUSTAL W : improving the sensitivity of progressive multiple sequence alignment through sequence weighting, position specific gap penalties and weight matrix choice. Nucleic Acids Res 22, 4673-4680.

Turner, S. L. \& Young, J. P. W. (2000). The glutamine synthetases of rhizobia: phylogenetics and evolutionary implications. Mol Biol Evol 17, 309-319.

Ueda, T., Suga, Y., Yahiro, N. \& Matsuguchi, T. (1995). Phylogeny of Sym plasmids of rhizobia by PCR-based sequencing of a nodC segment. J Bacteriol 177, 468-472.

Wang, E. T., van Berkum, P., Beyene, D., Sui, X. H., Dorado, O., Chen, W. X. \& Martinez-Romero, E. (1998). Rhizobium huautlense sp. nov., a symbiont of Sesbania herbacea that has a close phylogenetic relationship with Rhizobium galegae. Int J Syst Bacteriol 48, 687-699.

Wang, E. T., Rogel, A., Garcia-de los Santos, A., Martinez-Romero, J., Cevallos, M. A. \& Martinez-Romero, E. (1999a). Rhizobium etli bv. mimosae, a novel biovar isolated from Mimosa affinis. Int J Syst Bacteriol 49, 1479-1491.

Wang, E. T., van Berkum, P., Sui, X. H., Beyene, D., Chen, W. X. \& Martinez-Romero, E. (1999b). Diversity of rhizobia associated with Amorpha fruticosa isolated from Chinese soils and description of Mesorhizobium amorphae sp. nov. Int J Syst Bacteriol 49, 51-65.

Wernegreen, J. J. \& Riley, M. A. (1999). Comparison of the evolutionary dynamics of symbiotic and housekeeping loci: a case for the genetic coherence of rhizobial lineages. Mol Biol Evol 16, 98-113.

Xu, L. M., Ge, C., Cui, Z. \& Fan, H. (1995). Bradyrhizobium liaoningense sp. nov. isolated from the root nodules of soybeans. Int J Syst Bacteriol 45, 706-711.

Yang, G. P., Debellé, F., Savagnac, A. \& 9 other authors (1999). Structure of the Mesorhizobium huakuii and Rhizobium galegae 
Nod factors: a cluster of phylogenetically related legumes are nodulated by rhizobia producing Nod factors with $\alpha, \beta$-unsaturated N-acyl substitutions. Mol Microbiol 31, 227-237.

Young, J. P. W. (1985). Rhizobium population genetics: enzyme polymorphism in isolates from peas, clover, beans and lucerne grown at the same site. J Gen Microbiol 131, 2399-2408.

Young, J. P. W. (1992). Phylogenetic classification of nitrogenfixing organisms. In Biological Nitrogen Fixation, pp. 43-86. Edited by G. Stacey, R. H. Burris \& H. J. Evans. New York: Chapman \& Hall.
Young, J. P. W. \& Haukka, K. E. (1996). Diversity and phylogeny of rhizobia. New Phytol 133, 87-94.

Zhang, X. X., Turner, S. L., Guo, X. W., Yang, H. J., Debellé, F., Yang, G. P., Dénarié, J., Young, J. P. W. \& Li, F. D. (2000). The common nodulation genes of Astragalus sinicus rhizobia are conserved despite chromosomal diversity. Appl Environ Microbiol 66, 2988-2995.

Received 12 July 2000; revised 28 November 2000; accepted 15 December 2000. 